\title{
Aspectos rituales relacionados con el ganado en Santiago de Río Grande (II Región, Chile)
}

\author{
Reinaldo Lagos ${ }^{1}$, Emilio Mendoza ${ }^{1}$, \\ NOLbERTO AMPUERo ${ }^{1}$ y NORA HeRNÁNDEZ ${ }^{1}$
}

\begin{abstract}
RESUMEN
Se transcribe una entrevista con Emilio Mendoza, comunero del pueblo Santiago de Río Grande (comuna de San Pedro de Atacama), relativa a aspectos rituales del ganado. Se describe la solidaridad social a nivel de las contribuciones comunitarias como aporte en la formación de rebaños para con los jóvenes que inician su vida marital; se hace referencia a las creencias que norman la iniciación de la vida pastoril y a las ceremonias relacionadas con la prosperidad ganadera. Además, se hace especial mención de las divinidades asociadas con el ganado de los rituales de huaqui $y$ chuya $y$, de las ceremonias de tikavela $y$ pitira.
\end{abstract}

\section{ABSTRACT}

We transcribed the text of an interview with Emilio Mendoza, a resident of the pueblo Santiago de Rio Grande (San Pedro de Atacama) about the ritual relationships with livestock. We described the level of social solidarity by which the pueblo interacts in the formation of herds and animal husbandry practices. We make particular reference to the belief in these ceremonies of pastorial living and prosperous breeding. We also make special mention of divinities associated with the livestock, of the rituals of huaqui, chuya, and of the ceremonies of tikavela and pitira.

Cada vez que el hombre reconoce su pequeñez ante la magnificencia ambiental que le rodea y el usufructo que hace de los elementos naturales existentes, logra que se vacie la fuente interior depositaria de las virtudes o leyes del buen vivir -que con tanta frecuencia, ahí son olvidadas- produciendo en él una reacción o actitud alegre, confiada, sumisa y de dinámica mística, que motiva y abrillanta su humildad y su condición de ser un miembro más de la magia de la vida.

La clara visión que tiene el lugareño, componente de las pequeñas minorías étnicas de la Provincia de El Loa (II Región) de ser un ocupante temporal de la tierra, un aprovechador de los elementos y frutos naturales y de lo que le permite cultivar o desarrollar,

1 Miembros de la agrupación andina TOPAIN, CHILE. le lleva a establecer con ella contratos de compromisos espontáneos y personales de retribución en "pagos" u ofrendas y de nunca ir tras lo más, sino a lo estrictamente necesario, alejándose así de todo ambicioso egoísmo que altere la fluida convivencia con sus vecinos, con quienes contrae también la responsabilidad de respetar fundamentalmente al folklore que les caracteriza.

Justa exposición de este pensamiento y del hacer, es el llamado "enfloramiento del ganado", "floreo" o "poner aretes", hecho folklórico de gran dispersión geográfica nortina, sobreviviente del paso de los siglos y cuya claridad de su funcionalidad no necesita mayores explicaciones artificiales en la vida de los pobladores.

Contagiados con tal sencillez de los motivos, nos propusimos transcribir en una encauzada conversación, sus conocimientos sobre diferentes aspectos y rituales relacionados con el ganado, adquiridos básicamente en Santiago de Río Grande, pequeño poblado a $98 \mathrm{~km}$ de Calama, que por su condición geográfica mantiene más íntegra y menos contaminadas sus costumbres, como una técnica permitente de grata discusión, para más ágil comprensión de los lectores, adeptos al estudio del folklore de nuestra II Región.

\section{"Enfloramiento del ganado"}

Diálogo entre Emilio Mendoza C. y Reinaldo Lagos en Santiago de Río Grande, en el año 1983.

R.L.: Ciñámonos a lo que tengo como temario. Lo primero es: cómo se hace el hombre del ganado; cuáles son los medios para adquirir su ganado; si lo compra o lo recibe como herencia. Tomemos el caso de un matrimonio nuevo como base, ubicado más o menos a un año de formación.

E.M.: En primer lugar, todo comienza con el nacimiento de la niña o el niño, porque empieza con el bautizo u óleo que lo llaman, con el 
primer corte de pelo que tienen que hacer los padrinos, que justamente en esa oportunidad cuando se corta el pelo, los vecinos y familiares compran el pelo y otros le regalan al niño o niña, animales como patos, cabritos, ovejas, o llamitos.

\section{R.L.: Entonces esto viene a ser la primera adquisición...}

E.M.: Claro; que se llama herencia de pelo. De aquí depende que si el niño tiene o no suerte. Este corte de pelo es trabajo aparte pero como con él empieza la adquisición de animales, tomémoslo en cuenta. Cuando ya joven, se casa, entonces viene la segunda herencia, que es la herencia de los padres; ahí se les entrega los animales que ellos poseen, que fueron criados o asignados por los padres. Así se forma una pequeña tropa para el nuevo matrimonio que tiene que formar su nueva casa, de construir en los terrenos que también se le dan herenciales.

\section{R.L.: ¿Qué tipo de ganado es el más común...?}

E.M.: El principal es llamas, después ovejas y cabras; también vacunos que no es mucho, pero hay; el burro, que también es principal porque es el transporte junto con algún mular. Hay un orden jerárquico pero lo veremos más adelante.

R.L.: Veamos qué pasa una vez constituido el nuevo matrimonio y su salida a su nuevo hogar, con todo su ganado...

E.M.: Después del matrimonio o Likan santa o CharaChara, saldrán a ubicar su nuevo sector, para construir su propia estancia; su nueva casa, conforme a las reglas. Tienen que pensar muy bien cómo va a empezar a trabajar, cuál será su futuro, en otras palabras como nosotros decimos: sembrar para cosechar. Es decir, quiero llegar al momento en que ellos con todo el ganado que ya tienen en su poder, y que tendrán que seguir las costumbres como sus padres; tendrán que celebrar sus fiestas. Tendrán que hacer un compromiso con la tierra, tendrán que hacer un contrato, que quiere decir que liarán sus propias leyes según la tradición pero a sus ideas, no como lo hacían sus padres, poniendo sus propias costumbres, donde le quitan o ponen más costumbres; porque los enfloramientos son individuales. Ellos se comprometerán a servir a la tierra, a hacer todos los años los enfloramientos y señalamientos, para ir cumpliendo toda la vida, entonces para esto se abren canchas que son los pujgios en los diferentes lugares y diferentes rangos para cada ceremonia.

R.L.: Cuando ya van a salir del pueblo, ¿el hombre ya ha hecho su casa?

E.M.: No, porque como es nuevo matrimonio, tiene que salir recién a formalizar su estancia. En el caso de Río Grande, cada familia tiene determinado sector porque así está reglamentado por la Junta de Vecinos, entonces a un hijo también le corresponderá un sector. Para esto tiene que irse de inmediato para allá, porque no habrá nada hecho; tendrá que alojarse en el lugar y ponerse a hacer algo, el corral, la casa donde irán a vivir momentáneamente, hasta que haga algo efectivamente; digamos empieza todo allí.

R.L.: ¿O sea que construye prácticamente paravientos, para en seguida construir algo más definitivo?

E.M.: Exactamente.

R.L.: ¿Después viene ya la ayuda?

E.M.: En caso de la estancia, siempre la construye la persona sola. Para casa en el pueblo mismo, recibe la ayuda de los vecinos.

R.L.: Tomemos el caso de este matrimonio que va recién a salir ¿salen temprano?

E.M.: Todo se inicia con la salida del sol, para poder llegar hasta donde están destinados y tratar de formalizar rápidamente donde van a pernoctar. Ahora ellos mismos eligen los sectores porque deben ser uno de verano, de invierno, de otoño, o solamente dos. En cada sector tendrá que hacer su estancia.

R.L.: ¿Los acompaña alguien más?

E.M.: Difícilmente; a veces algunos familiares, no es común...

R.L.: ¿Llevan perros? 
E.M.: Eso sí. Tienen perros, como toda juventud es amante de criar sus perritos allá parten todos, perros, gatos, "con monos y petacas"; burros que los han heredado de sus padres, los animales de pelo que hayan tenido también, porque eso no es herencia de los padres, sino que es propiedad de uno mismo. ${ }^{2}$

R.L.: ¿A qué hora corresponde más o menos la salida del sol?

E.M.: Como a las 6 o 7. Depende donde esté también porque en el pueblo mismo llega tarde el sol...

R.L.: Mientras va caminando, supongamos que le corresponda tiempo lluvioso o de sol, en cualquiera de los dos casos ¿hay algo especial de lo que tenga que cuidarse?

E.M.: Depende de la época en que sea. Tendrá que partir para el lado que corresponda; si es para la parte baja que es para verano o para la parte alta que...

Otra voz: No, si es para la parte baja o serranía es para el invierno, porque si va para la parte alta es para el verano, pero comúnmente ocurren los casamientos en las fiestas principales, religiosas, generalmente están partiendo para la parte baja, para la fiesta del pueblo, es decir en invierno.

R.L.: Supongamos que mientras van caminando y los azota un rayo, ¿qué pasa?...

E.M.: Bueno, aquí supongamos que sea en verano y que van a la cordillera y si los azota o golpea un rayo, según lo que cuenta el pueblo, si el rayo cae adelante o atrás de todo el grupo, de aquí depende la suerte del matrimonio en su futuro, aunque esto puede suceder antes o después no precisamente durante el trayecto, pero supongamos que así fuera, esto indica que la naturaleza le está dando la oportunidad para guiarse o vivir; digamos si el rayo es de buena suerte ellos tendrán que "levantar" ese pujgio donde cayó el rayo, esa tierra, tendrán que llevarlo donde ellos van a construir el corral central, digamos allá deberán traspasarlo.

2 Referido a los animales recibidos como obsequio en el primer corte de cabello.
R.L.: Pero cuando cayó el rayo, ¿él lo toma así no más, o pide permiso para tomarlo?

E.M.: No, esto es oficial, no pueden tomar nada, porque tampoco podrán saber nada si es para castigo o no, con el paso de los días, tendrán que verlo con el yacho para que él diga lo que tienen que hacer, si es malo o es suerte. Si es suerte, el ganado será bienvenido porque ese pujgio tendrán que llevarlo al corral central o principal, donde van a celebrar las fiestas anualmente... y si es mala suerte, hasta ahí no más llegarán con el ganado porque se les terminará, y no tendrán que hacer nada... tendrán que inmigrar [emigrar] en este caso.

R.L.: ¿Y han ocurrido casos así...?

E.M.: Sí, han ocurrido casos tanto en los creyentes y no creyentes. Hay no creyentes que han dicho "bueno yo no creo en estas cosas de los antiguos, señalamientos, yo ahora sólo voy a vivir acá, yo compro corderos o de la herencia que tengo voy a hacerlos multiplicar y voy a vivir", pero resulta que eso no se da así porque la tierra le cobra sus derechos. Pachamama, que es la que reina en todo el ámbito, le cobra sus tributos de pastoreo y de uso, qué sé yo... ellos tienen que pagar y sin pago a la tierra no hay nada. Esto ocurre en todas las cosas, enfloramientos, señalamientos, siembras, en fiestas religiosas ...

R.L.: Entonces, él no toma nada hasta que lo haga un aysiri, para que éste haga el traspaso o traslado de la tierra...

E.M.: Claro, porque según la caída del rayo puede ser el mandamiento de una imagen, o de Pachamama, entonces el aysiri tendrá que leer cuáles son las órdenes para este matrimonio, los mandamientos, las obligaciones, qué es lo que la naturaleza les pide a ellos, porque si es buena suerte le está pidiendo muchas obligaciones que tendrán que cumplir con el tiempo, porque sabe que ellos son creyentes, son conocedores, son hijos de personas que han vivido haciendo ceremonias de pagos a la tierra, entonces la misma naturaleza les pide a través de ese castigo, que sería un castigo de la buena suerte. Entonces a través de lo que diga el aysiri contraerán obligaciones y más todavía, ellos pueden agregar cosas o quitar 
de otras ceremonias, o sea ellos lo arreglan a su modo, porque ellos como matrimonio van a ser los servidores.

R.L.: Supongamos que después de caído el rayo, pasó mucho tiempo, ¿cómo saben ellos, cómo ubican el sitio para hacer el traslado?

E.M.: Si cae el rayo es un accidente, puede matar todo o parte del ganado y si así fuera tendrían que devolverse al pueblo; si el castigo no es muy grande, entonces siguen adelante.

R.L.: ¿Existen otros peligros a los que se exponen?

E.M.: Hay varios, pero todo depende de él, porque si es creyente todo lo va a cumplir digamos, sin problemas y si no es creyente tenemos la de perder porque vendrán en castigo... si no hay un castigo de rayo estará la tierra misma, a través de Pachamama, a través de su perro principal que es el zorro, hará "cazuelar" todas las noches los corderos, a todos los llamos recién nacidos. El cóndor, el Mallku del aire, también mandado por Pachamama hará las suyas y así sucesivamente... el pujgio, el ojo de agua también cobrará su tributo, porque todas estas deidades, espíritus que viven, ellos cobran sus tributos, tanto el camino, el revolcadero, el cerro, el agua, el pasto, el monte. Todos cobran tributos, entonces ellos mismos van a liquidar ese asunto.

R.L.: Oye, ¿Tú dijiste pujgio de agua...?

E.M.: El manantial se respeta como un pujgio, no se traslada al corral, pero se respeta igual, en el mismo lugar.

R.L.: ¿Entonces un pujgio puede ser seco o con agua?

E.M.: Claro, con agua...

R.L.: ¿Pero el pujgio con agua recibe los mismos pagos que se le hacen al pujgio seco?...

E.M.: También se hace igual que todos los ritos ceremoniales, desde luego como corresponde a su rango. Vamos a una limpia de canales, también allí se ve el pujgio compuertas de agua, pero está la bocatoma, donde se entrega la ulpada...
R.L.: ¿Entonces la diferencia está en que no se le entrega el mismo tipo de cosas?...

E.M.: Exacto, dentro de su rango, por ejemplo, el cerro. El cerro tiene su cancha, si hay un pujgio, que nosotros podemos hacer, es decir, si un nuevo matrimonio puede hacer, es la costumbre de ir a ese sector, acostumbra de ir todos los años o cada vez que ellos llegan a ese lugar, ir a hacer una ceremonia en el cerrito más alto, eso ya constituye una pequeña canchita, porque allí ellos también harán un pujgio que respetarán como "sucursal" por todas las veces que hagan un enfloramiento. Porque esto es carta de presentación. Si nosotros nos vamos por ejemplo a Cortadera, que es la zona que nos corresponde, entonces iremos al cerro más alto que sería el Chujchul, allá iremos a hacer los huaqui con la familia y se hace toda la ceremonia momentáneamente, dando aviso para que todas las deidades protejan al ganado. Después cuando ellos se van del sector, también van a despedirse allá en el cerro y en el corral, porque en el corral también, cuando hacen un rodeo, también cada vez que se llega y cada vez que se vayan tendrán que hacer una ceremonia para poder alojar y como despedida.

R.L.: ¿Cuánto tiempo se demorarían más o menos en llegar al sitio que les corresponde?

E.M.: Normalmente un día solar, pero a veces también corresponde dos días.

R.L.: Si tuvieran que viajar de noche, ¿podrían aparecer los espíritus, encarnados por ejemplo en un zorro, un león?

E.M.: Claro, pero no cuando van estas personas en buenas condiciones; digamos el peligro acecha cuando van en contra de las costumbres.

R.L.: Volviendo otra vez al caso de la caída de un rayo, la mala suerte vendría si cayera adelante, porque si cae atrás del ganado, ahí no ocurriría nada, salvo que cayera en el medio del grupo...

E.M.: Claro, porque llega a quemar la cola, o el centro, queda la matanza, mueren 10 o 20 o 30, puede morir hasta la persona, aunque esto ocurre, pero supongamos que si pasara, si logra a "pescar" a una persona y esta muere 
y si el acompañante queda vivo, esta persona no puede hacer nada por el caído, ni moverlo, sino que dejarlo ahí, irse al otro día, porque así como un rayo lo mató puede caer otro rayo entre las 24 horas y éste lo hace revivir. Al vivir esta persona, ya no es la misma, porque queda con poder... Aquí es donde nace un Aysiri, un yacho; porque un yacho nace así o se hace; hay dos posibilidades, así los que son tirados del rayo, son los de más poder, en la parte del bien, son de derecha.

R.L.: Cuando el matrimonio llega ya al sitio donde se van a instalar, ¿qué es lo primero que hace? ¿pide permiso a la tierra?...

E.M.: Lo primero que hace es hacer un huaqui de bienvenida, después de instalarse momentáneamente. Ubica una mesa momentánea, es decir, un tendido de una llijlla o una manta, saca los implementos, la tinca; la chuspa de coca, echa licor en un par de vasos, sea vino, chicha o aloja, y como solamente están los dos, hacen la ceremonia, pidiendo lo que ellos necesitan y seguidamente el hombre toma los dos vasos y sigue caminando por la derecha, contra el reloj, alrededor del lugar que ellos están, va a escanciar hojas de coca y licor para luego regresar al mismo lugar de partida y así tomar hacia el naciente y como los vasos los lleva con la mano izquierda, con la derecha va sacando hojitas y tirando a los alrededores; pero cuando llega a la parte naciente, coge los vasos con la mano derecha, un vaso, y lanza el licor hacia la salida del sol, siempre por la derecha, no como Serracino ${ }^{3}$, lo hizo en la tele, por la izquierda, al revés. Después hace el cambio de vaso y tira el otro porque éste es caso especial en que hay un solo varón, entonces tiene que hacerlo así, cuando hay más varones, más huaqui, van tres, cuatro personas, depende.

R.L.: A ver, ordenemos esto, porque falta aclarar mucho la ceremonia, la ubicación de la mesa y orden del proceso:

$1^{\circ}$ Bendice las cuatro esquinas de la mesa que está ubicada de tal manera que cada lado dé

3 George Serracino, ex sacerdote maltés e Investigador del Museo Arqueológico de San Pedro de Atacama. hacia un punto cardinal. Cada esquina representa una estación de ciclo anual. El hombre oficiante se coloca en el lado opuesto al que dé hacia el este, es decir tras del lado oeste de la mesa. Así la esquina $n^{\circ} 1$ es la que queda a su derecha y corresponde a la primavera, que se inicia para los riograndeños, el $1^{\circ} \mathrm{de}$ agosto, junto con el nacimiento del Carnaval y comprende los meses de agosto, septiembre y octubre.

La esquina $\mathrm{n}^{\circ} 2$ está entre el sur y el este y corresponde al verano, meses de noviembre, diciembre y enero.

La esquina $n^{\circ} 3$, queda entre el norte y el este, representando al otoño, meses de febrero, marzo y abril.

La esquina $n^{\circ} 4$, entre el norte y el oeste, corresponde al invierno, meses de mayo, junio y junio. Este último mes es el de las fiestas patronales del pueblo.

Inicia la bendición con la bebida, escanciando $o$ chispeando sobre la esquina $\mathrm{n}^{\circ} 1$. Enseguida sobre la ${ }^{\circ} 2,3$ y 4 , hablando en voz alta o en silencio, pidiendo que se cumplan sus aspiraciones o deseos, completando así lo que ha pedido desde el momento en que fue echando la coca al vaso con bebida.

$2^{\circ}$ Enseguida con el mismo vaso (depende del tipo de bebida que él tenga en esta ocasión, pues oficialmente deben ser tres tipos de bebidas: chicha de maíz, chicha de algarrobo (aloja) y vino), inicia una rueda grande (caminando de derecha a izquierda) escanciando y nombrando los lugares que estarán relacionados con su vida pastoril, pidiendo a ellos para que le entreguen lo mejor.

$3^{\circ}$ Una vez completada la vuelta o rueda, vuelve a salir pero esta vez (siempre por esquina $n^{\circ} 1$ ) directamente hacia el naciente (en línea recta). Ahí, en un punto determinado por él, lanza todo el contenido del vaso, haciendo un movimiento semicircular, como un arco, de derecha a izquierda con el brazo (movimiento contrario a las manecillas del reloj). Ya realizado esto, hace una reverencia y observa si es que ha quedado algo en el vaso, y si así sucede, ello es un indicio que las tierras no están muy "hambreadas" (es como una 
satisfacción para el ofrendante). En caso que no haya quedado nada en el vaso, significa que habrá que hacer muchos pagos, para dejar conforme a las deidades a través del tiempo en que él viva en el lugar. En el mismo lugar e instante, al ver el resultado en el vaso, saca sus conclusiones respecto al significado de lo observado y sin hacer comentario en voz alta, retorna al sitio inicial en la mesa, conservando la dirección derecha a izquierda. Mientras camina y en caso que hayan quedado hojitas adheridas al vaso, las coge cuidadoso con los dedos y las va dejando caer por el trayecto. Al llegar a su lugar, recién habla diciendo en voz alta las conclusiones que sacó. Sirve más bebida en el vaso y lo ofrece al aire en forma de brindis con frases relatoras de sus deseos. Al libar la bebida, analiza el sabor, suavidad o aspereza de ella ya que, según como sean estas cualidades, servirán para reafirmar las conclusiones que ya ha sacado.

Una vez realizado el huaqui o pago a la tierra, vuelve a echar bebida en el vaso para, esta vez, ofrecerla a su esposa y ella brinde libremente, ya que no es su obligación de hacer el proceso puesto que éste, en la ocasión, es privativo del hombre.

R.L.: Hemos visto que la mesa puede ser de tipo común o si es que no ha llevado, ser reemplazada por una llijlla, que tendida en el piso cumple tan función. Pero, ¿dónde la ubica? ¿cómo elige el sitio para ponerla?

E.M.: Las personas que van por primera vez, buscan o ya tienen visto un lugar o zona que sea refugio contra el viento, que esté siempre con el sol de la mañana o que esté atrás de una colina. La mesa que se ubica puede ser en cualquier lugar, porque es momentánea, pero después, cuando ya construya su corral ahí, pondrá la mesa en sitio definitivo, siempre hacia el naciente.

R.L.: Es conveniente aclarar bien sobre qué es un huaqui, pues un tipo sería el que ya hemos estudiado con la llegada de este nuevo matrimonio.

E.M.: Claro, ese sería uno. Yo le voy a dar un ejemplo como huaqui. Vámonos un poco íntimo, particular; supongamos que vamos a hablar de un conjunto de música, supongamos que como sikuris vamos a visitar a don Reinaldo en su casa; nosotros vamos a visitarle, conversar con él y entonces él -como dueño de casa, si es que así lo quiere- nos va a brindar una tinca por nuestra visita y si esta persona, dueño de casa, quiere que la tinca que nos sirve, nosotros le hagamos un huaqui, entonces estamos autorizados para hacerlo. Pero cuál sería el inicio del gesto; la persona dueña de casa conjuntamente con las bebidas él quiere un huaqui porque "yo quiero que hagan alcanzar, hagan el pago para mi casa", entonces junto con las bebidas, el vino, la aloja o la chicha vendrá la uncuña o las chuspas, es decir viene la coca, y nosotros como músicos sin voz de mando viendo que viene la coca, tendremos que hacer inmediatamente el huaqui, entonces tender la mesa; ¿ah?, ¿estará la mesa en el patio o no habrá mesa? y si no hay mesa entonces se usa el bombo como tal, para salir del paso, pero inmediatamente el director de música dice: "bien, músicos, vamos a hacer alcanzar el huaqui del dueño de casa”. Ahí empieza un pequeño huaqui...

R.L.: A ver, ahí hay un detalle, porque dices tú... Estábamos claros cuando decías que este proceso es el huaqui, pero ahora dices "vamos a hacer alcanzar el huaqui" o sea, van a hacer alcanzar la tinca, ahí se está refiriendo a la tinca.

E.M.: Sí, a la tinca...

R.L.: ¿Por qué entonces esa confusión?

E.M.: El huaqui mismo puede ser una tinca... si el deseo del dueño de casa me trae una botella y la coca, entonces vamos a hacer un huaqui; si el dueño de casa me trae tres tinca, de las tres tengo que hacer tres huaqui vamos a tener que hacerlas alcanzar, ocupando al director de los músicos y otras personas, nosotros vamos a tener que hacerlas alcanzar; vamos a ser tres portadores de huaqui...

R.L.: ¿O sea, no es que se le llame huaqui a la chicha o al vino, sino que es a lo que se va a formar de la unión?

E.M.: Efectivamente, el huaqui sería el encuentro del vino o bebidas con la coca en el vaso. Es lo mismo que ocurre con el huaqui de sangre; 
en que se recoge la sangre en un recipiente de cerámica y se le echa la coca, pasando a constituir un huaqui de sangre. Es lo mismo acá, en que el dueño de casa quiere un huaqui, entonces él me va a traer la coca, entonces vamos a servir a echar el vino en un jarrito y vamos a invitar inmediatamente a los músicos a hacer el huaqui, cada uno...

R.L.: En ese caso, ¿con vino sería un huaqui de vino?

E.M.: Huaqui de vino...

R.L.: ¿Y si fuera con chicha...?

E.M.: Sería un huaqui de chicha.

R.L.: Un huaqui de chicha, es decir la unión de ésta con la coca...

E.M.: Un huaqui de aloja. Para el caso también en limpia de canales constituye la ulpada o agua con harina, viene a ser un huaqui también..

R.L.: Bien, pero como ahora, el huaqui, es decir, esta unión del vino o de la sangre con la coca, según el caso, del líquido con la coca, más claro.

E.M.: Constituye huaqui...

R.L.: ¡Ya! eso constituye un huaqui que complementa, que encierra a su vez lo que se va a hacer con eso, con el destino que se le va a dar a esa unión de coca con bebida?

E.M.: Exactamente, exactamente...

R.L.: Entonces, la unión de los dos elementos, más el proceso que se va a realizar, es el huaqui...

E.M.: Exactamente. En el caso de los músicos al dueño de casa, se va a hacer un huaqui según las bebidas que él traiga, para la salud del dueño de casa, para la casa misma, o sea, se va a hacer un pago íntegro hacia los moradores y la casa.

R.L.: En el caso de este matrimonio que hace su primera mesa al llegar, esta mesa improvisada, digamos, el huaqui es sólo para la Pachamama, lo que encierra desde luego a él y no para las almas, es decir, no entrega al oeste.
E.M.: Como ésta es la primera vez, lo hace para las deidades, pero cuando él haga su primer "enfloramiento", en la víspera recién lo hará para las almas, en que tendrá que quemar algunos elementos. ${ }^{4}$

R.L.: Para este matrimonio que llegó a ocupar su lugar, el huaqui es una petición de permiso?

E.M.: Sí, una petición de principio, de permiso para la instalación...

R.L.: Entonces, ¿no es un sahumerio?

E.M.: No.

R.L.: ¿Es lo mismo que un pago?

E.M.: Sí.

R.L.: O sea, él estaría haciendo una petición de permiso a través de un principio de pago?

E.M.: Pero, puede suceder también que pueda ser un tipo de sahumerio con $c o a,{ }^{5}$ pero en el caso, es todo imprevisto, es muy sencillo ...

R.L.: ¿Qué pasa con el ganado? Para dormir, después de haber cumplido el pago, ¿se acuesta su mujer al lado de él, juntos con el ganado?...

E.M.: Bueno, ellos tienen que dormir, digamos cerca del ganado siempre encajándolo hacia un alero de un peñasco, de un cerro y dormir a la "perspectiva" de que no se vayan o lleguen animales que vayan a cazarlos, como zorros...

R.L.: Es decir, “perspectiva”... ¿cómo estar alerta?

E.M.: Alerta.

R.L.: Ahora, veamos qué ocurre esa noche, puesto que es la primera vez que están solos; el primer encuentro que tienen ambos con el medio ambiente, con la pampa misma...

4 En la "noche de los abuelos"; ver Lagos y colaboradores (1982).

5 Coa Santiago, es un tipo de hierba que se usa para sahumar. 
E.M.: Bueno, aquí podrían haber razones, pero la noche transcurre y siempre como ellos llevan un perrito también, tal vez podrán dormir, tal vez no... yo creo que la sensación debe ser bastante grata creo yo, puesto que se encuentran por primera vez solos y en la oscuridad de la noche... y al cuidado del ganado... bueno, los comentarios pueden ser muchos a imaginarse, pero la vida empieza aquí medio dura para ellos, creo yo...

R.L.: Bueno... pasó la noche... supongamos que durmieron bien, ¿con los rayos del sol despiertan?

E.M.: Al alba.

R.L.: Eso es antes de la salida del sol?

E.M.: Sí.

R.L.: ¿Qué pasa allí?

E.M.: Bueno aquí ya empiezan a movilizarse. Lo primero habrá que levantar la cama, preparar los alimentos y... si hay animales chicos, hay que hacerlos mamar. Bueno... la mujer tendrá que, después de un buen desayuno-almuerzo, tendrá que partir al cuidado del ganado, buscar los sitios de pasto y el hombre tendrá que dar comienzo a los cimientos de la casa-estancia, digamos del corral mismo.

R.L.: La señora se va con el ganado al sitio que ella cree conveniente... ¿cómo elige éste? ¿Tiene que ser algo especial?...

E.M.: Bueno, tiene que partir para cualquier lado... buscando el mejor pasto o el mismo ganado tira para el mejor pasto; van descubriendo nuevas áreas de pastizales, de desfiladeros, de caminos, rinconales, de lomas, etc. ...

R.L.: Supongamos que llegó a un pasto bueno ¿pide permiso, hace algo especial o lo ocupa simplemente?

E.M.: No, no se hace ninguna cosa especial, solamente se toma como pastoreo, por zona; van combinando eso sí, van en rotativo porque no todos los días pueden comer en el mismo sector, entonces va rotando digamos, a la redonda.
R.L.: Una vez que ella ya logró su función, ¿a qué hora más o menos regresa?

E.M.: La salida de la estancia se hace más o menos, para el buen pastor, en el caso del nuevo matrimonio, si es que son activos, se hará muy temprano, ahora si son ellos medio dejaditos, medio regalones, empezarán tarde porque se ha dado ocasión en que a veces son las 11:00 del día y el ganado todavía no sale del corral, siendo que debe salir no mucho tardar de las 08:00 de la mañana. Entonces, ellos empiezan temprano, ya a la salida del sol tienen más o menos preparado. Toman el desayuno-almuerzo, a las 8 ú 8:30 están partiendo al cerro, para bajar más o menos a las 3 de la tarde, por decir, 3, 4, ya están cayendo para el corral o estancia.

R.L.: Al quedarse solo el hombre, ¿a qué se dedica de preferencia?

E.M.: De preferencia, hay que tomar de base... señalizar el corral; las piedras debe movilizarlas, para que el ganado se ubique, darle forma...

\section{R.L.: ¿Cuadrangular o circular?}

E.M.: De los dos gustos... Enseguida tendrá que construir el techo, el techo de la casa misma, de la casa-cocina, al lado del corral. Para ello buscará o aprovechará el alero de una roca o una cueva y si no hay nada de esto, tendrá que poner las piedras para hacer el techo, $\mathrm{y}$ después con el tiempo ya va concluyendo el corral...

R.L.: Ahora veamos el "enfloramiento" mismo. ¿Para qué se hace el "enfloramiento"?

E.M.: Es bastante complejo el asunto porque reúne tantas situaciones de ceremonias rituales y qué sé yo, para... con el único fin de hacer los pagos correspondientes a la tierra.

R.L.: ¿A la tierra o a los animales o a los cuidadores de los animales, o a los dioses?...

E.M.: En todo, para los animales, para la tierra, para las deidades, en conjunto, para todos... porque aquí se sabe más o menos que el animal para vivir, tiene que comer, para beber tiene que ir 
a un manantial a tomar agua, para dormir tiene que tomar un terreno, entonces este animal tiene que pagar ese consumo que ha hecho durante el año, a los pastizales, a la tierra, a los manantiales, entonces, se le va a enflorar al ganado, a él, para que haga alcanzar el pago a quién deba.

R.L.: ¿Significa que esto de alcanzar, es llevar el mensaje, traspasar?

E.M.: Exactamente, traspasar, es decir, al animal se le colocan las flores de lana en el cuerpo y en las orejas, se le escancea vino en su cabeza, en su panza, chicha, aloja, coca, todo, para que él vaya a su respectivo paraje donde él come, descansa o bebe agua, qué sé yo, porque sería muy difícil creo yo, que el dueño vaya por cada uno de los lugares en que anduvo el ganado durante el año; no tendría tiempo...

R.L.: Tiene que ver con los patronos del pueblo también el "enfloramiento"?

E.M.: También está relacionado, por el ganado, porque sabemos que el llamo tiene como patrono a San Antonio, las cabras a San Bartolomé, las ovejas a San Juan, los burros a San Ramón, los caballos a San Santiago, los vacunos a San Lucas.

R.L.: ¿Entonces, al "enflorarlos” también se les está...

E.M.: También se le debe hacer partícipes a los santos patronos, es decir, llevándoles unas velas o haciéndoles unas misas, que quizás no sea en la misma oportunidad pero siempre más antes o después, porque tiene que estar presente el nombre.

R.L.: Si una persona extraña a los vecinos que están participando llega a la ceremonia, ¿qué significado tiene para los dueños del ganado?

E.M.: Los dueños del ganado cuando van a hacer la fiesta, invitan a todo el pueblo, es decir, se sabe quiénes van a llegar, pero de vez en cuando ocurre que van otras personas junto con los vecinos, que son ajenos a las invitaciones y ajenos también a los pobladores, estas personas son recibidas en forma especial aunque sean vecinos que no fueron invitados, todos son recibidos en forma especial pasando a ocupar lugar especial en la mesa porque estas personas pasarían a representar a una deidad, ya sea a un cerro, un volcán, a un camino, a un manantial, etc. No pueden ser rechazados...

R.L.: Los nombres de los dioses más comunes son la Pachamama, Coquena que es la parte masculina de la Madre Tierra, qué otros nombres hay?

E.M.: Claro, también están, claro que ya son secundarios, están: el viento, agua, el revolcadero, el volcán, el cerro, el camino, después se ocupan a los cerros porque cada cerro tiene un nombre especial o representa una suposición de una deidad territorial.

R.L.: ¿Y la coca?

E.M.: También.

R.L.: ¿Pero no tienen nombres especiales?

E.M.: No, yo no los conozco.

R.L.: Y ¿cómo les llaman a los dioses? Mallkus?

E.M.: En general se le llaman: las deidades; pero los Mallkus pasan a ser representados por los cóndores, porque se supone que los cóndores van más allá de las tierras, que son difíciles de alcanzar por intermedio del ganado o de las personas mismas, entonces, los cóndores vuelan más allá, a otras vidas que habrán en los montes.

R.L.: ¿En qué fecha se hace el "enfloramiento”?

E.M.: El "enfloramiento" es libre; la fecha la fijan los dueños del ganado, pueden concluir con el día del santo patrono del ganado o generalmente entre los meses de diciembre, enero, febrero, o también puede ser el 2 de febrero, también el "jueves comadre" o el "jueves compadre", que son antes del Carnaval o el "sábado Carnaval" mismo o el "lunes de Carnaval". Más acá de estas fechas no puede pasar, porque después del "jueves tentación" todo tiene que concluir.

R.L.: ¿Y en qué consiste esto del "jueves comadre" y "jueves compadre"? Hace tiempo que 
estamos viviendo eso y todavía no hemos sacado nada.

E.M.: Todavía no hay claridad al respecto.

R.L.: Dejémoslo por el momento, pero hay que seguir estudiando el hecho. Sigamos con: ¿en qué lugar territorial se hace el "enfloramiento"?, ¿en la estancia o en el pueblo?

E.M.: Eso depende del dueño del ganado; a veces toma como corral principal en la casa del pueblo; en otras oportunidades toma el lugar en las zonas altas en la cordillera, especialmente para el verano, entre los meses de diciembre a febrero.

R.L.: ¿Y qué pasa con los vecinos que ayudan con el "floreo"?

E.M.: No hay ningún problema; los que tienen voluntad van a ayudar; van igual pues han sido invitados en la fiesta anterior y en otras fechas; ellos lo saben.

R.L.: Tienen algún significado especial las "flores" con las que adornan a los animales?

E.M.: Claro; tenemos las "flores" de lana de corderos, de lana de llamos, plumas de avestruz, de algodón, de lana de cunte.

R.L.: ¿Qué es cunte?

E.M.: Lana de alpaca, pero teñida de un color "concho de vino".

R.L.: ¿Eso sirve para todos los animales?

E.M.: Dentro del rango sí. Para los corderos es lana de llama; para llamos, lana de cordero; para cabras, lana de cordero o llamo; el burro lleva flores o adornos de plumas de avestruz, la alpaca no se conoce mucho, pero si se enflorase sería con lana de cordero; los caballos y los vacunos con bozales de hilos de lana de cordero y llamo; también usan bozales los chivatos, carneros y el burro marucho (padre de la manada).

R.L.: Las flores, ¿son en forma de copihues?
E.M.: Claro, las flores son como pompones de lana, sujetados con hilos y amarrados o cosidos a las orejas, lanas sobre el lomo.

R.L.: ¿Cuáles son los llamados “ojitos”?

E.M.: Son ... se llaman flores de "conchas". Dentro del ganado ovejino cabrío, hay cabras con orejas largas, con orejas caídas y con orejas cortas "concha", o "cuchara". Entonces en estas de cuchara o concha tiene forma especial, se le ponen los llamados ojitos o flores de algodón teñido y bien apretado.

R.L.: ¿En el “enfloramiento”, las personas se adornan con flores?

E.M.: Claro, esto ocurre en "enfloramiento" y "señalamiento", viene "enfloramiento" en un día especial, al final del "floreo" se le va colocando a cada participante una florcita en el vestón o sombrero, pero en "señalamiento", se le marca también al visitante, queda marcado con las marcas del dueño del ganado, en el pecho o en la camisa.

R.L.: Para realizar el "floreo", ¿cómo se junta al ganado? ¿se hace rodeo?

E.M.: No. Primero viene el "campeamiento", que es la junta del ganado total. No puede quedar ningún animal olvidado o perdido.

R.L.: ¿O sea es la concentración del ganado en un punto y después viene el rodeo?

E.M.: Claro, el rodeo es para llevarlos al corral, después de haberlos juntado a todos.

R.L.: ¿En el "campeamiento”, quiénes participan?

E.M.: Toda la familia participa en la búsqueda de corderos, llamos, burros, etc.

R.L.: Durante el "campeamiento" y rodeo, ¿hay algún peligro a que están expuestos?

E.M.: Peligros no, pero problemas grandes, sí. Por ejemplo hay veces en que no se encuentra; que se pierde una tropilla de llamos o de cabros, que por más que se busquen, no se encuentran, esto significa que el cerro los tiene cubiertos en alguna parte y no los deja que los dueños 
los vean. Quizás sea por una falta de pago; hay algún problema. Sucede también que a veces de tanto buscar, por ejemplo 15 días, buscando una llama con su guagua, hecho ocurrido en la vida real, han dejado la llama, olvidada ya, por ser imposible de encontrar. En esa ocasión se centraron en la zona de Lagunitas para el "enfloramiento", con toda la tropa ya, menos la llama con su guagua, pero el día antes del floreo, en la víspera, fueron a rodear el ganado y la llama había llegado hacía segundos ¿con su guagua? Viajó siguiendo la tropa... pero ¿dónde estaba?... ¿por qué no se le encontró?... Ese problema tendrá que aclararlo el aysiris que estará encargado de los problemas del "enfloramiento", conversará con los espíritus de los cerros.

R.L.: La separación del ganado, ¿se hace en el mismo corral, o al llegar al corral?

E.M.: Todo el ganado está junto, junto pero no revuelto, tanto llamas como cabras, ovejas, burros, etc., pero sí en corrales paralelos...

R.L.: ¿O sea, están separados por muros?

E.M.: Los animales de ganado mayor se acomodan cerca del corral o del manantial; los corderos, cabros y llamos se guarecen en el corral. El día de la víspera ahí sí que se encierran todos en el corral.

R.L.: ¿Cómo se elige el guía de la tropilla?

E.M.: No puedo asegurarlo, pero se le da la oportunidad al macho. El dueño del animal, cuando ve que éste tiene condiciones, lo deja, es decir, no lo mata. Además selecciona por raza.

R.L.: ¿En un "enfloramiento” cuál es la obligación de los dueños de casa?

R.M.: Hacer los pagos a los dioses.

R.L.: ¿Y con las visitas, qué atenciones debe hacer?

E.M.: Las atenciones son: la amistad y tincas especiales. Es atendido con tincas en la mesa central.

R.L.: ¿Tiene que prepararse en forma especial?
E.M.: No. El dueño de ganado está preparado; él sabe lo que tiene que gastar, sabe lo que va a invertir, sabe a lo que va, porque tiene que cumplir los pagos correspondientes a como contrajo el compromiso con la tierra; hablemos del mismo matrimonio que ya está viviendo tres o cuatro años, entonces tiene que hacerlo tal como él se comprometió al principio con su contrato verbal con la tierra. Si se olvida, para eso está el yacho que tiene que recordarle lo que debe pagar a la tierra.

R.L.: ¿En qué consiste el compromiso con la tierra?

E.M.: Los compromisos pueden ser por ejemplo, que yo me comprometo ante la tierra de servirle a ella para que me dé mayor porcentaje de ganado, mayor multiplico.

R.L.: Pero ¿con qué cosas o cómo le vas a servir?

E.M.: Ya. Por ejemplo. Yo digo. Asiento de pujgio en el corral; ese pujgio yo lo voy a servir con corazón vivo de un cordero blanco macho, acompañado con pajas especiales ... ¿уya? ... ese sería un punto; el sacrificio del animal, en la mañana, al alba, en la salida del sol, como sea mi compromiso... y, pero... anterior a esto tengo que hacer en la noche la tikavela, tengo que hacer el recorrido por las zonas cercanas, tengo que ir al cerro más alto, donde tengo un pujgio de enlace, de sucursal; tengo que ir allá y de allá anunciar, un día anterior al alba o al mediodía, según... De allá voy a anunciar, con flautas y banderas, voy a decir; voy a hacer el "enfloramiento", vengan todas las deidades, la invitación es para todos. El asiento para las deidades y mallku son monedas de peso fuerte (plata).

R.L.: ¿En ese momento está solo?

E.M.: Solo o acompañado con la señora, depende como él haya pactado el acuerdo. Y si yo quiero hacerlo allá solamente con flautas y banderas, con huaqui o una invitación general, ese va a ser mi cumplimiento. Ahora si yo, voy, digo... por primera vez aquí sacrifico un cordero y echo sangre a todas las deidades que vengan, la invitación es como un aperitivo, entonces voy a tener que hacerlo todos los años en que voy a hacer "enfloramiento", o 
depende, si en "señalamiento", según como esté hecho el pacto.

R.L.: Este pacto lo hizo ¿cuándo?

E.M.: Al primer año ...

R.L.: ¿Al cumplir el año?

E.M.: No, al primer año, todavía no cumple el año, al primer año... Cuando hablamos nosotros del primer huaqui, del rodeo para hacer su casa. Entonces cuando él hizo su casa... hizo su corral... no tiene cancha, no tiene pujgio... entonces ahí va a formar su pujgio, va a abrir una cancha... a lo mejor le cayó un rayo... él va a llevar su pujgio para allá y si no va a tener que hacer un pujgio... con ceremonias, con todo, completo, completo; él va a conversar con su señora... va a hacer una costumbre nueva... no va a tomar las ceremonias del suegro o del papá porque él tiene otras costumbres más complejas o menos complejas... seguramente parte va a traer de ahí y aquí lo va a agregar más de su parte... a él le gusta así, le gusta asá. El le va a agregar más cosas a su gusto, a su manera pero eso tendrá que cumplirlo durante los años de su vida...

R.L.: Cuando hace eso, ¿ahí él invita a un Aysiru?

E.M.: Claro, ahí sí pues...

R.L.: ¿Y puede invitar a otros vecinos?

E.M.: Puede invitar a cualquiera...

R.L.: Pero... generalmente, ¿invita?

E.M.: Claro, invita.

R.L.: ¿Entonces allí explica a todos o explica al pujgio no más?

E.M.: Al pujgio no más, el compromiso es con la tierra, no con la gente...

R.L.: Ahí, él dice voy a cumplir, a hacer los pagos, todos los años, o cada tantos años, voy a hacer una entrega de tal cosa, en estas condiciones... mientras yo viva estaré haciendo esto y esto...
E.M.: Claro, estaré sirviendo a la tierra... Ahí entonces, por ejemplo, él dice: Yo me comprometo... para todos los "señalamientos"... a la gente que venga al floreo le regalo un cordero, eso ya está en el contrato, entonces toda la gente que va a ese "señalamiento", él les tiene que regalar un cordero de su multiplico...

R.L.: ¿“señalamiento” y “enfloramiento”?

E.M.: Claro, "enfloramiento" y "señalamiento"... Digamos, los "enfloramientos" se hacen, anualmente, pero los "señalamientos" son cada cuatro años, tres o cinco, depende como sea el multiplico de la tropa, entonces tiene que señalarlos, marcarlos... eso es paralelo, la ceremonia del contrato para "enfloramientos" es para una parte y concluye en otro programa más amplio para el "señalamiento"... entonces dentro de eso juega el papel... resulta que el dueño de repente se olvida y no cumple con sus compromisos y entonces hay una falta... no sacó el corazón, no entregó el corazón o no llevó el cordero pa'l cerro como tenía que llevarlo todos los años... entonces eso constituye una falta...

R.L.: La tikavela es la responsabilidad que tiene que cumplir en el día anterior al "enfloramiento"... ¿cuál es la tikavela misma?

E.M.: Hablemos de tikavela y tikallijlla... La tikallijlla es la llijlla con flores... y la tikavela son las flores completas la llijlla con flores $\mathrm{y}$ las velas, se hace un velatorio.

\section{R.L.: A ver, veamos eso...}

E.M.: Claro, si hablamos de cuando la llijlla sola, es llijlla... pero con flores adentro ya viene a ser la tikallijlla, es decir llijlla con flores pero ese mismo conjunto viene a ser tikavela cuando se hace el velatorio de las flores, sería...

R.L.: ¿Y las velas dónde se colocan?

E.M.: En la mesa.

R.L.: ¿Sobre las llijllas?

E.M.: Sobre o al lado de las llijllas. 

R.L.: Porque la llijlla está cubriendo la mesa, ¿no es cierto?

E.M.: Hay dos llijllas. Una cubre la mesa y la otra que cubre las flores...

R.L.: Hum... entonces... La tikavela significa que sobre la llijlla que cubre la mesa se pone otra más y que sobre ésta en su centro están colocadas las flores. Ahora, las puntas de la llijlla que tiene las flores son dobladas para tapar las flores?

E.M.: Para tapar las flores... pero se descubren...

R.L.: ¿Cuándo?

E.M.: En el momento del acto, es decir no solamente una tikallijlla... hay tikallijlla con flores para cabras y corderos, una tikallijlla con flores para los llamos...

R.L.: O sea, ¿hay varias en la mesa?

E.M.: Exacto, y una tikallijlla con flores para los burros, es decir están aparte...

R.L.: Es como hacer varios montoncitos. O sea si son cinco tipos de animales, habrá cinco llijllas con sus flores propias según el tipo de animal sobre la llijlla general.

E.M.: Con sus cosas correspondientes a usar dentro de la ceremonia del "floreo".

R.L.: Ahora, esas tikallijllas... pasan a ser tikavela...

E.M.: En el momento del velatorio.

R.L.: ¿Colocan las velas alrededor de cada llijlla?

E.M.: Alrededor de los tres bordes prácticamente, donde están los dueños, los yachos, visitas.

R.L.: ¿Y cuántas velas se usan?

E.M.: De acuerdo a los santos patronales que se deba ofrendar.

R.L.: ¿Cuántos pueden ser más o menos?
E.M.: De uno puede ser hasta 12 velas, porque aquí van de acuerdo a los propietarios del ganado ... porque si en la tropa hay cuatro, o 10 propietarios, entonces los 10 van a colocar las velas para los santos patronos, que son como cuatro o cinco patronos...

R.L.: ¿Es decir para el patrono de las cabras, llamos...?

E.M.: Exacto, del pueblo... y caballos. Ahora cada familia... tienen un caballo o un mular... no todas pero siempre los hay.

R.L.: ¿Sobre qué se acomodan las velas?

E.M.: Palmatorias de cerámica, platitos. Estas velas... eh... el proceso es el siguiente: con mucha anticipación el dueño del ganado las lleva a la iglesia, las pone al pie del patrón, encomendándose ya de lo que va a hacer... y que serán encendidas allá en la mesa, en su mesa, en la tikavela al nombre del ganado... y lo lleva en esa noche de velatorio.

R.L.: ¿A cuál patrono?

E.M.: A los patronos, pues... puede ser a San Bartolo...

R.L.: ¿En el caso de que este pastor tenga cinco tipos de animales?

E.M.: A los cinco.

R.L.: ¿Entonces pondrá una vela, o un paquete de vela por cada uno ante cada patrono?

E.M.: Claro... porque si él tiene ovejas, cabros, caballares, llamos, burros, si no está cumpliendo con uno.

R.L.: Tú recién ibas a decir otra cosa y yo te interrumpí...

E.M.: Claro, de acuerdo como estábamos conversando, el dueño del ganado se anticipa con tiempo, a veces el compromiso es que las velas se prendan algunas en la mesa del tikavela y las otras son devueltas nuevamente para prenderlas en la iglesia... pero esto como digo queda sujeto al tipo de costumbre que va a hacer el dueño... 
R.L.: ¿Se consume toda la vela?

E.M.: Se consume toda.

R.L.: ¿O sea, se hace en la noche... quedando encendida hasta que acabe?

E.M.: Toda la noche, hasta que se termine la vela.

R.L.: ¿Hay cuidadores o personas que se quedan para el velatorio?

E.M.: Como el trabajo es lento y van ya muy atrasados, entonces en la noche del velatorio, las visitas o las señoras, hombres y mujeres, van preparando las flores para el ganado, lo van construyendo en ese momento, porque está el material y no está hecho, entonces tienen que acelerar... a pedido del dueño de casa ellos tienen que trabajar... entonces, ahí transcurre parte de la noche... ahí se termina... van a dormir pero alguien queda cuidando... queda con la guitarra; queda entrenándose con el instrumento... en fin...

R.L.: Volvamos, Emilio, al momento especial en que el pastor está en el cerro para comunicar que realizará el "enfloramiento"...

E.M.: Concurre el dueño del ganado a veces acompañado, a veces no... depende como sea el compromiso, pero junto a todos los elementos que él necesita lleva una pluma de loro... pluma de loro hablador, para que esta pluma de loro hablador sea la que traspase la invitación oficial a todas las deidades... claro que todos no usan esta pluma de loro, pero esto es, como digo, para hacer más efectivo la festividad.

R.L.: ¿Y qué hace con la pluma de loro?

E.M.: El lo deja en el pujgio...

R.L.: La deposita no más.

E.M.: Exactamente...

R.L.: ¿Al depositarla significa que la pluma va a hacer su trabajo?

E.M.: Eso, exactamente.
R.L.: ¿Y ... las deidades llegan al "enfloramiento"?...

E.M.: ¡Llegan! [es categórico E.M.], llegan porque a través de un forastero, a través de personas que no fueron invitadas van a llegar "mimetizados" con estos personajes. Para esto entonces, en la mesa central del corral, dentro de la tikallijlla con flores está una uncuña... Una uncuña con hojas de coca y aquí... frente, en la misma posición de las autoridades, del yacho... se van colocando monedas de plata... monedas de plata con el cóndor a la vista (monedas antiguas)... entonces se van colocando las monedas de acuerdo a las invitaciones que haya... estas monedas son puestas ahí... éstas son asientos para las deidades. Entonces ahí está la mesa reservada, para las deidades pero el cuerpo presente pasa a las visitas que vienen y se sientan...

R.L.: Tú dices que en la tikallijlla, sobre la uncuña... estas monedas se colocan en cada grupo, porque hablamos que habían cinco o más grupos.

E.M.: Pero es que esto ocurre en el día del "floreo"...

R.L.: ¡Ya! o sea que va a estar la tikallijlla perteneciente al tipo de animal que se va a "enflorar"... solamente?

E.M.: Claro.

R.L.: ¿Las monedas son colocadas de acuerdo a la posición que tiene el hombre hacia el este? ¿Delante de él?

E.M.: Exactamente, delante de él...

R.L.: A ver, ordenemos el proceso de la "víspera"... Primero va el hombre al cerro más alto a invitar a las deidades.

E.M.: Claro... A la oración de la tarde o de la noche, procede a hacer la pitira... la pitira se realiza con todo el ganado en el corral, donde van todos los participantes acompañados de los instrumentos musicales... "dentran" por la derecha, acompañando al yacho, es decir aquí la música juega un papel muy importante porque parece que cubre todo lo que se está haciendo 
en la intimidad, tapa todo lo que está haciendo el yacho... el yacho con su ayudante va...

R.L.: Espera un poco... ¿quieres decir como una cortina protectora contra malos espíritus?

E.M.: Contra los malos espíritus... porque hay un "poupourri" de música... la guitarra toca sus sones de "enfloramiento", el tambor con su voz ronca suena y suena, y la flauta con melodías de carnaval que va por la montaña o cerros y después baja... la anata y si hay charango también va tocando y si hay arpa también suena pero cada instrumento suena dentro de su música... desvirtuando total, lo que se está haciendo.

R.L.: ¿O sea que no es un solo tema el que se va tocando...? ¿cada músico va tocando algo diferente, nada que ver con el resto de los músicos?

E.M.: Claro... diferente en el mismo momento... pero música solamente, no canto.

\section{R.L.: ¿No copleando?}

E.M.: ¡No!... entonces aquí la "bandera” va adelante, va la coa... (reemplaza al incienso), va la tikallijlla en brazos... van los ayudantes, los músicos, los acompañantes, el pastor, la "bandera" que va adelante haciendo o abriendo camino, echando el ganado para el centro del corral para que no quede nadie fuera de donde van a pasar el yacho y los demás... siempre echándoles pa'l centro, juntándolos, reuniéndolos totalmente, el ganado en el corral... entonces aquí como que el músico cumple con el papel de cubrir el trabajo que ya está haciendo el yacho, porque está centralizado para hacer la pitira... va cortando lana... primero corta "chuya" y luego corta lloque... La chuya son lanas de hilos de vicuña, la chuya es para la derecha, el lloque... es torcido a la izquierda de hilo blanco con lana negra... es para los muertos.

\section{R.L.: ¿La chuya de qué color es?}

E.M.: La chuya es color de vicuña, es decir color natural... entonces él va pitirando... se demora en hacer el trabajo... da como nueve vueltas... tres y tres.
R.L.: Cómo ... ¿ ¿tres y tres?... ¿por qué?...

E.M.: Porque así tiene que ser...

R.L.: ¿Va pasando delante de cada animal?

E.M.: Por todos los animales...

R.L.: Pero... ¿tiene que dar nueve vueltas dices tú?

E.M.: Puede ser tres, seis o nueve ...

R.L.: ¿Pero con la chuya no más?

E.M.: Con la chuya, con la pitira, con el lloque... con las tres cosas...

R.L.: A ver... la pitira ¿cuál es entonces?

E.M.: Es el conjunto en total de lo que se tiene que hacer con el ganado...

R.L.: O sea... ¿dar la vuelta e ir juntando el ganado?

E.M.: No... la pitira es todo, todo lo que se va a hacer dentro del corral... pero dentro de eso viene primera vuelta... la chuya... el cortar los hilos... los hilos los va cortando en forma de cruz en cada cabeza del ganado... digamos el yacho cumple aquí el papel de limpiar el ganado... pedir lo bueno... de la salud, del pastoreo, sanearlo... lo mismo con el lloque... que va en la parte final, para los muertos, para sacarles el alma...

R.L.: Espera... volvamos atrás otra vez... Cuando te refieres a las tres vueltas... eso de ir chuyando... ¿ ies otra cosa me parece?

E.M.: Es chuyar con agua de resto de chicha, es decir, cuando se hace la chicha, el dueño hace un combinado de la cocción del arrope, viene juntamiento se puede decir... para la fermentación de la chicha... antes de eso se separan las aguas de la harina, no estoy seguro cómo se hace... pero ahí como que se asienta el harina de maíz, entonces el agua se aflora y es casi transparente, se junta y se guarda para esta ceremonia... y la otra, el resto se deja para 
chicha. Entonces, hablando de chuya, tenemos la chuya-passia y la chuya de chicha.

R.L.: ¿Cuál es la chuya-passia?

E.M.: Una chuya es con harina de maíz y la otra chuya es la chuya-passia que se extrae del tubérculo de la sicha, que crece en las raíces de la brea, chilca y legía y este tubérculo que florece y deja flor, se saca los granos. Esos granos se secan y se muelen y esa harina pasa a formar la parte de la chuya.

R.L.: Sicha, entonces, se llama la planta. ¿Es cultivada o crece natural?

E.M.: Es silvestre, es comestible.

R.L.: ¿Es como una papa?

E.M.: Es como una papa hija de otra papa... y esa papa viene a ser como un tubérculo ajeno a las raíces de la brea.

R.L.: Bien, ahora dime ¿con esa chuya-passia va trabajando el yacho?

E.M.: Claro, esa chuya-passia se junta con la agua de chuya que es de la chicha... porque la otra chuya va a ser de agua simple con harina de "maíz negro", "maíz culle"... entonces tenemos una chuya para la derecha y la otra que es para la izquierda, para los muertos, que es agua de "maíz negro".

\section{R.L.: ¿Y la passia?}

E.M.: La passia es la que se usa para la derecha... con la chuya... agua y chuya de chicha, ¿entiende?

R.L.: No, me enredé...

E.M.: Ya... a ver...

R.L.: ¿Serían tres tipos de chuya?

E.M.: No, dos no más... es que el otro lleva el nombre de chuya, no más... el agua de chicha es como el nombre del agua no más... viene a ser chuya pero sin nada.
R.L.: A ver... si es como lo entiendo... hay un depósito con la chicha de maíz... el agüita pura de arriba se tomó y se mezcló con la otra que es de la sicha. Esto pasa a formar una chuya oficial que es para la derecha.

E.M.: Y la otra es de agua... que es con harina de "maíz negro", "culle", que es para la izquierda.

R.L.: ¿Esa chuya es... la harina, esa mezclada así no más con agua o se dejó algún tiempo a remojar?

E.M.: No, así no más.

R.L.: O sea, ¿es como hacer la ulpada no más?

E.M.: Claro, más o menos...

R.L.: Y esa agüita... ¿el concho se deja?

E.M.: No... todo, todo... Lo mismo que la otra se echa así en conjunto y se va tirando...

R.L.: Chuya...

E.M.: Chuya, chuya de chuya-passia.

R.L.: Que es una sola...

E.M.: Sí, una sola.

R.L.: Formada por dos elementos.

E.M.: Eso...

R.L.: ¿Y la otra?

E.M.: Chuya de "maíz blanco".

R.L.: Chuya de maíz negro.

E.M.: Ese va de "maíz negro" con "maíz blanco", según...

R.L.: Una es chuya-passia y la otra chuya de maíz.

E.M.: Chuyando se hacen tres vueltas de corral.

R.L.: Chuyando, ¿es decir va rociando con la...? 
E.M.: Con las dos chuyas... paralelamente... con las dos... una el ayudante y otra el yacho... el ayudante va para cumplir... va echando de la otra...

R.L.: Con la mano derecha echando.

E.M.: Con la derecha va tirando.

R.L.: ¿Tirándolo al aire?

E.M.: Sí, tirándolo... para el ganado, pa'l cerro, para los dueños de casa.

R.L.: Cuando se le acabó eso... ¿le pasa otro vaso?

E.M.: En el mismo vaso.

R.L.: ¿Lo toma con la izquierda ahora?

E.M.: Claro...

R.L.: ¿Por qué, ahora va a tirar para las almas?

E.M.: Sí, para las almas... Entonces, aquí también viene problema porque a veces... todo depende como esté planificado, si el programa del dueño está hecho primero para la derecha... hace las tres vueltas a la derecha... después tres vueltas a la izquierda... entonces depende como esté planificado... a veces junto.

R.L.: Allí hace tres vueltas no más entonces...

E.M.: Claro, tres vueltas no más...

R.L.: ¿Las tres vueltas es para completar, que no se le escape nada?

E.M.: Exacto... Aunque en una sola vuelta haya cubierto todo... Después vienen las tres de "lana chuya".

R.L.: Entonces, primero chuyando hizo tres vueltas... Con una chuya o con la otra haría seis... y después vienen las tres vueltas recién de corte de lana que es para la derecha primero y también llamada chuya...

E.M.: Lana chuya, lana para la vida.
R.L.: Veamos cómo es lo que dices del corte en cruz sobre la cabeza del animal.

E.M.: Para esto el dueño del ganado hiló un ovillo de lana, entonces el yacho lo lleva en sus alforjas y de allí lo saca para ir cortando...

R.L.: Cuando el yacho va cortando... tiene el ovillo con la mano izquierda y la punta con la mano derecha... Entonces, estira un trozo de unos 15 centímetros sobre la cabeza del animal, es decir traza el brazo central de la cruz... luego, tira fuerte con la derecha y haciendo sonar al tiempo que se rompe el hilo (lana). Enseguida, traza la parte transversal (otro brazo de la cruz) y de la misma manera... con sonido, corta la lana. Esto lo va repitiendo muy rápidamente sobre cada animal que se presente por delante en forma casual, sin que se lo vayan poniendo... Esta operación la hace tantas veces pueda en cada una de las tres vueltas...

E.M.: No es necesario que deba hacerlo con cada uno de los animales, sino que al que le toque... pudiendo tocarlo las tres veces al mismo animal o ninguna vez.

R.L.: Mientras va cumpliendo esto, ¿va hablando?

E.M.: De acuerdo al compromiso va pidiendo, va limpiando de todos los males...

R.L.: El resto de la gente ¿qué hace en ese momento?

E.M.: Acompañan solamente, en fila como comitiva... mientras él está haciendo, la música va sonando, va cubriendo todo...

R.L.: ¿En fila o en "rueda”...?

E.M.: Otra "rueda".

R.L.: O sea que todos dan las tres vueltas.

E.M.: Claro.

R.L.: ¿Y los dueños de casa?

E.M.: Van juntos... van llevando las cosas, la tikallijlla, los elementos. 
R.L.: Terminadas las tres vueltas de la chuya, ¿sigue de inmediato con las otras?

E.M.: Inmediatamente... no se estacionan... porque todo lo llevan en la mano... porque la mesa está en la sala, aquí todo es portátil...

R.L.: ¿El ayudante y las personas se encargan de portar?

E.M.: El ayudante, dueño de casa... todos.

R.L.: ¿De dónde se inicia el recorrido?

E.M.: De la puerta principal del corral.

R.L.: Esto fue para la derecha, ¿qué pasa con el lloque?

E.M.: Siguen el mismo movimiento por la derecha.

R.L.: ¿Cambia solamente la mano del yacho?

E.M.: La mano y la lana... viene blanco y negro...

R.L.: ¿O sea tira con la izquierda, haciendo la cruz igual?

E.M.: Siempre lo mismo... devolverse no se hace nunca...

R.L.: Una vez hechas las tres vueltas... ¿qué más viene?

E.M.: Terminan concluyendo y haciendo el anuncio oficial que el "enfloramiento" se va a hacer en el día de mañana...

R.L.: Espera un poco... todavía no me parece claro... Entonces las nueve vueltas significa que: primero pasa chuyando con chuya-passia y chuya de maíz negro... Las tres siguientes con lana...

E.M.: Pero mejor pongámosle, don Reinaldo... una cada una mejor, porque si no se va a hacer un lío... o sea 12 vueltas.

R.L.: Entonces, primero sería chuya-passia... llevamos tres... después chuya de "maíz negro"... y llevamos seis. Después chuya con lana para la derecha... serían nueve y después las tres vueltas con lloque ¿correcto?

E.M.: Exacto.

R.L.: Ahora, dime, ¿qué es lo que sigue a toda la pitira...?

E.M.: Con esto queda el ganado, oficialmente que el día siguiente se va a hacer el "floreo"... es decir... el ganado hasta esta parte no sabía qué se iba a hacer.

R.L.: ¿Terminado en el corral se dirigen a la casa?

E.M.: A la casa... pero antes de esto vamos a concluir... el yacho no botó ninguno de los pedacitos de lana que fue cortando en las alforjas, en las cuales las echa separadas. No estoy seguro... pero algunos usan algunas pajas en este mismo proceso... sería como acompañar la chuya y el lloque... Resulta que al final de esto, salen los yacho, porque en las alforjas lleva todo preparado, lleva cuchillo, fósforos, lleva tinca, lleva "contras"... saliendo del corral él con su ayudante se va... a un sector ajeno, mientras los músicos pasan a la casa... entonces se despistan las personas del yacho... y éstos se van a una quebrada, cerca de la casa donde las lanas de vicuña, de la derecha lo van a enterrar y el otro, el de la izquierda lo va a quemar.

R.L.: Entonces es lo mismo que pasa en la "noche de los abuelos".

E.M.: Entonces él lleva su tinca, de chicha o vino y hace la ceremonia particular y hace la entrega oficial a la Pachamama y a las ánimas...

R.L.: ¿Después regresa a la casa?

E.M.: Claro, y ahí viene un descanso porque mientras se ha hecho la pitira, los familiares han preparado la mesa para la comida. Entonces, cuando ellos llegan... ubican en otra mesa que tienen especial, las llijllas, las tikallijllas mientras van a la cena, a la merienda... llega el yacho y su ayudante... pasan a ocupar su lugar especial... 
R.L.: Bueno, me parece que hasta acá llega esta conversación, aunque estoy consciente que es mucho lo que falta por decir de este complejo folklórico; sin embargo, lo discutido es muy importante para satisfacción de nuestros objetivos.
E.M.: Claro que sí. Falta mucho, pero en otras conversaciones o trabajos van a ir saliendo otras cosas... así iremos completando.

\section{GLOSARIO DE TERMINOS}

ALOJA: Chicha al algarrobo (Prosopis sp.).

AYSIRI: Adivino, yacho, persona con poderes para interpretar acontecimientos o indicios ocultos y predecir el futuro. Intermediario entre las deidades y lugareños.

BREA: Arbusto (Tessaria absinthioides).

CAMPEAMIENTO: Reunión, concentración, agrupamiento de todo el ganado disperso en campo.

CANCHA: Lugar abierto donde se encuentra el pujgio.

COMPROMISO: Contrato, promesa o pacto espiritual con la tierra de continuar las tradiciones de acuerdo con las ideas de la persona que lo hace.

COQUENA: Deidad campesina. Parte masculina de la Pachamama.

"CON MONOS Y PETACAS": Referente a irse con todos los objetos que se posee.

COA: Arbusto seco (Lephidophyllum cuadrangulare, Mey), para sahumerio. Se conoce también como "coba", "chacha", "cova", "con Santiago".

CUNTE: Lana de alpaca (Lama pacos) teñida de color concho de vino, burdeo.

CHARA CHARA: LIKAN SANTA: Fiesta de casamiento o cumpleaños.

CHILCA: Arbusto (Género Baccharis o Fluorensia Campesiris).

CHUYA: Esta voz tiene varias acepciones:

a) Como sustantivo al referirse a la mezcla de agua de la chicha con harina de maíz.

b) También para nombrar el hilo de lana de vicuña torcido para el lado derecho.

c) Como sinónimo de escanciar.

d) Fermento dulce que queda luego de preparada la chicha.

CHUSPA: Bolsa pequeña tejida finamente en lana, para portar hojas de coca.

CHUYA PASSIA: Bebida resultante de mezcla del agua de la chicha con harina de los granos de la flor de la "siena".
ENFLORAMIENTO: Complejo ceremonial que concluye con el prendimiento de "flores de Lana" en las orejas, cuello y lomo de los animales.

ESCANCIAR: Salpicado de bebidas y otros, con los dedos, en direcciones y objetos determinados, durante ceremoniales.

ESTANCIA: Habitación en el campo, usada para actividad pastoril.

FLOREO: Acción de prender las "flores de lana" en los animales.

GANADO: Referido a grupos de animales. Conjunto.

HUAQUI: Voz con tres acepciones:

a) Ceremonia de pago, de agradecimiento o rogativa a las deidades.

b) Unión de la o las bebidas o sangre con hojas de coca, de ofrecimiento ceremonial.

c) Como "tinca".

UNCUÑA: Paño cuadrangular de 20 a $30 \mathrm{~cm}$ por lado aproximadamente, tejido con lana. Sobre éste se colocan las hojas de coca.

LAGUNITAS: Lugar al norte de Santiago de Río Grande. Cercano a Caspana.

LEGIA, LEJIA: Arbusto (Bacharis tola) de hojas encrespadas.

LLIJLLA - LLICLLA: Manta de lana de un metro cuadrado aproximadamente; de uso múltiple. No puede faltar en el atuendo personal de las mujeres. Los hombres también la usan. Normalmente se le lleva sobre la espalda.

LLOQUE: Hilo de lana de color blanco con negro y torcido hacia el lado izquierdo. Es entregado como ofrenda a las ánimas.

MALLCU - MALLCO: Deidad a la cual representa el cóndor.

MARUCHO: Nombre que se da al animal considerado como "padre" de la manada entre asnos y caballares.

MULTIPLICO: Resultado de la reproducción del ganado.

PACHAMAMA: Máxima deidad, "madre tierra", "santa madre tierra", de tipo dual con Coquena, ella es la parte femenina. 
PARAVIENTO: Pircado o enramado bajo de protección provisoria contra el viento.

PITIRA: Ceremonial que se hace en el corral para espantar los males y lograr perdonamiento. Consiste en cortar "chuya" y "lloque" sobre la cabeza de los animales.

PUJGIO: Orificio hecho en la tierra de más o menos 25 a $30 \mathrm{~cm}$ de profundidad y $15 \mathrm{~cm}$ de diámetro, el cual es cubierto por una piedra plana. En él se depositan todas las ofrendas y tincas dedicadas a la Pachamama. Representa a la "Santa Madre Tierra”.

RODEO: Conducción del ganado hacia el corral principal para realizar "enfloramiento" o "señalamiento", una vez efectuado el "campeamiento".

RUEDA: Desplazamiento que se hace caminando en círculo por el contorno interior del corral y alrededor de la mesaaltar. Es parte principal en la coreografía de danzas rituales $\mathrm{y}$ festivas.

SEÑALAMIENTO: Marcación del ganado cada tres o cinco años en ceremonia conjunta con el "enfloramiento".
SICHA: Tubérculo subterráneo silvestre (Juelia subterránea). Es comestible.

TIKAVELA: Velatorio de las "flores de lana" en la noche anterior al "enfloramiento" y en la "noche de los abuelos" (Lagos et al. 1982).

TIKALLIJLLA: En la llijlla con los elementos necesarios para hacer las "flores de lana" o que contiene a éstas y que serán usadas en el "enfloramiento".

TINCA: Bebida alcohólica ofrecida como voluntad en ceremoniales y fiestas comunes.

TROPA: Totalidad del ganado que se posee. Rebaño.

ULPADA: Bebida de harina tostada, mezclada con agua.

YACHO: Curandero; intermediario entre deidades y el lugareño; hombre sabio. Existen rangos o categorías como curandero de derecha y de izquierda, curandero de salud, de hechicerías (librar de ellas y hacerlas), de almas, como consejeros.

\section{REFERENCIA CITADA}

LAGOS, R., E. MENDOZA, N. AMPUERO y N. HERNANDEZ,

1982. "La noche de los abuelos en Santiago de Río Grande".

Chungara 9: 247-274. 